\title{
Implementation of Telemedicine for Urgent and Ongoing Healthcare for Patients with Parkinson's Disease During the COVID-19 Pandemic: New Expectations for the Future
}

\author{
Esther Cubo ${ }^{\mathrm{a}, \mathrm{b}, *}$, Anhar Hassan ${ }^{\mathrm{c}}$, Bas R. Bloem ${ }^{\mathrm{d}}$ and Zoltan Mari ${ }^{\mathrm{e}}$ on behalf of the \\ MDS-Telemedicine Study Group \\ ${ }^{a}$ Department of Neurology, Hospital Universitario Burgos, Burgos, Spain \\ ${ }^{\mathrm{b}}$ Department of Health Science, University of Burgos, Burgos, Spain \\ ${ }^{\mathrm{c}}$ Department of Neurology, Mayo Clinic, Rochester, MN, USA \\ ${ }^{\mathrm{d}}$ Department of Neurology, Radboud University Medical Centre, Donders Institute for Brain, Cognition and \\ Behavior, Centre of Expertise for Parkinson and Movement Disorders, Nijmegen, The Netherlands \\ ${ }^{\mathrm{e}}$ Cleveland Clinic Lou Ruvo Center for Brain Health Department, Las Vegas, NV, USA
}

Accepted 5 May 2020

Due to the COVID-19 pandemic, many countries have taken drastic measures to slow down infection rates. These include physical and social distancing, and in some countries, a lock-down of non-essential business and marked restrictions on social and economic life [1]. These measures, while necessary to contain the pandemic, do come with particular concerns around the increased vulnerability of the many patients living with one or more chronic diseases, including Parkinson's disease (PD) and other movement disorders [1]. Thus far, coronaviruses have not been linked to specific long-term neurological sequelae on patients with PD [2]. However, recent literature discusses the possibility of an increased risk for cerebrovascular disease due to the severe inflammation

\footnotetext{
*Correspondence to: Esther Cubo, MD, PhD, Neurology Department, Hospital Universitario Burgos, Spain. Avda Islas Baleares, 3, 09006, Burgos, Spain. Tel.: +34 947256533/ Ext 35380; E-mail: mcubo@saludcastillayleon.es.
}

associated with COVID-19 [3]. In addition, recent studies indicate that alpha-synuclein participates in the innate immune response to any viral infection, and the intriguing observation of anosmia associated with COVID-19, a common feature of prodromic PD, might just represent a coincidence, but warrant further studies $[2,4,5]$.

The earliest healthcare change during this time of crisis has been to limit access to clinics and neurology wards to preserve fragile PD and other movement disorder patients from becoming infected. In some regions, the shortage of medical staff has forced movement disorders neurologists to provide care for COVID-19 patients instead [6]. Hence many patients with PD and other movement disorders are likely to benefit from restored access to subspecialty care via telemedicine, whether this is videoconferencing or simple telephone consultations. Also, even after the immediate threats of the current COVID-19 outbreak have been brought under control, we will likely be 
facing a need for continued restrictions on public and social life for months or even years to come, until a vaccine is found.

Implementation of telemedicine for the delivery of urgent and ongoing healthcare has rapidly scaled upwards [1]. Many neurologists and other health professionals are currently using a variety of different telemedicine healthcare tools at their disposal to continue delivering patient care. Telemedicine tools include simple phone calls, use of e-mails or text messages, and video visits. Telemedicine can, therefore, be used for routine follow-up, urgent visits, new subspecialty consultations, research visits, psychotherapy, genetic counseling, social services, rehabilitation, and education. Telemedicine is not superior to the quality of care delivered with regular in-person visits, but it is associated with comparable outcomes, and offers greater efficiency and service for patients. The merits and benefits of telemedicine are supported by a small but growing body of evidence [7-10]. However, telemedicine has yet to be established universally for virtual management of device aided therapies in PD and other movement disorders, which will require the additional technological implementation of a secure remote digital interface within deep brain stimulation and infusion pump devices [11, 12].

In order to assist movement disorders neurologists worldwide, the Movement Disorders Society (MDS) Telemedicine Study Group has created a "step-bystep" guide [13], including specific requirements for reimbursement and regulation, incorporating the latest information available in several countries and global regions. The Telemedicine Study Group have posted an educational webinar to reflect recent telemedicine changes related to the unfolding COVID-19 pandemic, and how to set up a successful Movement Disorders telemedicine practice [14]. In addition, the Telemedicine Study Group also has developed a network of regional experts covering the globe to continue to provide updated information as telemedicine guidelines continue to evolve. In this regard, a web form to post questions is available on the MDS website [15]. Continuously updated regulatory information and guidelines, and a robust Q\&A section addressing all relevant questions posted by MDS members [16]. We hope to hear about the hands-on experience with telemedicine from many colleagues in the field, as this will help to further shape optimal delivery of telemedicine services for patients, and holds great promise of becoming a routine part of working in the future.

\section{TELEMEDICINE STUDY GROUP}

Jamie Adams, Mitra Afshari, Zakiyah Aldaajani, Jason Aldred, Jawad Bajwa, Hilla Ben-Pazi, Patrick Browne, Adriana Cardozo, Bill Chan, Sylvain Chouinard, Jacques Doumbe, Marieke Dekker, Shinsuke Fujioka, Nicholas Galifianakis, Emilia Gatto, Christopher Goetz, Mark Guttman, Maya Katz, Jaime Martin, Emile Moukheiber, Oluwadamilola Ojo, Njideka Okubadejo, Alexander Pantelyat, Ali Shalash, Meredith Spindler, Caroline Tanner, Jinyoung Youn.

\section{CONFLICT OF INTEREST}

Prof. Bloem currently serves as co-Editor in Chief for the Journal of Parkinson's disease, serves on the editorial of Practical Neurology and Digital Biomarkers, has received honoraria from serving on the scientific advisory board for Abbvie, Biogen and $\mathrm{UCB}$, has received fees for speaking at conferences from AbbVie, Zambon, Roche, GE Healthcare and Bial, and has received research support from the Netherlands Organization for Scientific Research, the Michael J Fox Foundation, UCB, Abbvie, the Stichting Parkinson Fonds, the Hersenstichting Nederland, the Parkinson's Foundation, Verily Life Sciences, Horizon 2020 and the Parkinson Vereniging.

No other authors have a conflict of interest to report.

\section{REFERENCES}

[1] Helmich RC, Bloem BR (2020) The impact of the COVID19 pandemic on Parkinson's disease: Hidden sorrows and emerging opportunities. J Parkinsons Dis 10, 351-354.

[2] Papa SM, Brundin P, Fung VSC, Kang UJ, Burn DJ, Colosimo C, Chiang HL, Alcalay RN, Trenkwalder C, the MDS-Scientific Issues Committee (2020) Impact of the COVID-19 pandemic on Parkinson's disease and movement disorders. Mov Disord Clin Pract, doi: 10.1002/mds.28067

[3] Victorino DB, Marcia Guimarães-Marques M, Nejm M, Scorza FA, Scorza CA (2020) COVID-19 and Parkinson's disease: Are we dealing with short-term impacts or something worse? J Parkinsons Dis, doi: 10.3233/JPD-202073

[4] Rey NL, Wesson DW, Brundin P (2018) The olfactory bulb as the entry site for prion-like propagation in neurodegenerative diseases. Neurobiol Dis $\mathbf{1 0 9}$ (Pt. B), 226-248.

[5] Tulisiak CT, Mercado G, Peelaerts W, Brundin L, Brundin P (2019) Can infections trigger alpha-synucleinopathies? Prog Mol Biol Transl Sci 168, 299-322.

[6] Fasano A, Antonini A, Katzenschlager R, Krack P, Odin P, Evans AH, Foltynie T, Volkmann J, Merello M (2020) Review management of advanced therapies in Parkinson's disease patients in times of humanitarian cri- 
sis: The COVID-19 experience. Mov Disord Clin Pract, https://doi.org/10.1002/mdc3.12965

[7] Srinivasan R, Ben-Pazi H, Dekker M, Cubo E, Bloem B, Moukheiber E, Gonzalez-Santos J, Guttman M (2020) Telemedicine for hyperkinetic movement disorders.Tremor Other Hyperkinet Mov (N Y) 10, doi: 10.7916/tohm.v0.698

[8] Ben-Pazi H, Browne P, Chan P, Cubo E, Guttman M, Hassan A, Hatcher-Martin J, Mari Z, Moukheiber E, Okubadejo NU, Shalash A; International Parkinson and Movement Disorder Society Telemedicine Task Force (2018) The promise of telemedicine for movement disorders: An interdisciplinary approach. Curr Neurol Neurosci Rep 18, 26.

[9] Schneider RB, Biglan KM (2017) The promise of telemedicine for chronic neurological disorders: The example of Parkinson's disease. Lancet Neurol 16, 541-551.

[10] Achey M, Aldred JL, Aljehani N, Bloem BR, Biglan KM, Chan P, Cubo E, Dorsey ER, Goetz CG, Guttman M, Hassan A, Khandhar SM, Mari Z, Spindler M, Tanner CM, van den Haak P, Walker R, Wilkinson JR; International Parkinson and Movement Disorder Society Telemedicine Task Force (2014) The past, present, and future of telemedicine for Parkinson's disease. Mov Disord 29, 871-883.
[11] Zhang C, Zhang y, Zhan S, Li D, Jin H, Denys D, Sun B (2018) Telemedical deep brain stimulation: Merits and limitations. Stereotact Funct Neurosurg 96, 272-273.

[12] Zhang C, Li D, Zeljic K, Tan H, Ning Y, Sun B (2016) A remote and wireless deep brain stimulation programming. Neuromodulation 19, 437-439.

[13] https://www.movementdisorders.org/MDS/About/Committ ees-Other-Groups/Telemedicine-in-Your-MovementDisorders-Practice-A-Step-by-Step-Guide, Accessed April 26, 2020.

[14] https://www.movementdisorders.org/MDS/About/Committ ees-Other-Groups/MDS-Study-Groups/TelemedicineStudy-Group, Accessed April 26, 2020

[15] https://form.jotform.com/200906888114054, Accessed April 26, 2020

[16] https://www.movementdisorders.org/COVID-19Pandemic-MDS, Accessed April 26, 2020. 